\title{
The Computing of the Influence of a Steady Element on the Polarization Spectrum
}

\author{
R. Cimbala, I. Kršňák, I. Kolcunová
}

This paper describes computation of the influence of a steady current element on the current response of insulation material. The steady element is always present. When the insulation is dry, it can be neglected. When moisture is present, the steady element has to be calculated. A decision can be made after examining the spectrum.

Keywords: polarization spectrum, insulation system, current response, time of stabilization, steady element.

\section{Introduction}

The insulation strength of a material can be scanned only by observing the changes in the electro-physical structure of the material without destroying it. The breakdown voltage can only be determined by performing an electrical strength test. This is a destructive test on an insulation system made of solid material. This test is not suitable for service conditions [1].

The relations between the quantities are evident. Changes in material structure due to service ageing necessarily change the material properties and electrical strength. In this way the determination of quantities closely connected to an electrophysical structure requires the quality of the material to be specified [2].

Many evaluation methods can be used to determine the state of an insulation material. There is no single method at present that can provide a full description. A set of methods has to be used. Some of them involve observing the polarization spectrum. As Fig. 1 shows, the polarization spectrum is quite wide.

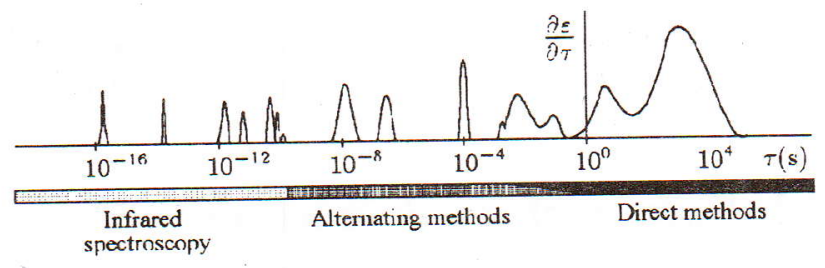

Fig. 1: Common polarization spectrum

The available methods range from infrared spectroscopy to direct methods. Most information can be found between $10^{-4} \mathrm{~s}$ and $10^{+5} \mathrm{~s}$. This is the field where current or voltage responses can be observed mainly. There are two way to access the polarization spectrum [3].

\section{Two ways of accessing the polarization spectrum}

The area of interest is in the range between $10^{-4} \mathrm{~s}$ and $10^{+5} \mathrm{~s}$. We decided to monitor the polarization spectrum by means of current responses. Absorptive or resorptive current is a macroscopic exhibition of the polarization processes inside the material [4].

\subsection{Macroscopic exhibition}

Analysis of the polarization spectrum of the insulating material is nowadays based on measuring the charging and discharging processes [5].

A method based on applying direct voltage will be described below. Direct methods are based on observing current or voltage time responses. Well-known methods are polarization indices, absorptive or resorptive current analysis, recovery voltage or self-discharge analysis. There is no problem with power supply, as in the case of the alternating diagnostic method. For example, when measuring a capacitive object with a capacity of $10 \mathrm{nF}$ and applied voltage $10 \mathrm{kV}$ the requisite current is about $100 \mathrm{~mA}$. A powerful supply is needed.

When applying external direct voltage forming an internal electrical field inside the material, the total current shown in Fig. 2 consists of the following components. While charging with direct voltage current from the geometrical capacity, absorptive and steady current are present. After some time the material is discharged, but only two currents are present resorptive current and current from the geometrical capacity. Steady current is not present because there is no external electrical field.

$$
i_{t}(t)=i_{c}(t)+i_{s}+i_{a}(t)
$$

$i_{t}(t) \quad$ total current,

$i_{c}(t)$ geometrical capacity current,

$i_{a}(t) \quad$ absorption current,

$i_{s} \quad$ steady current.

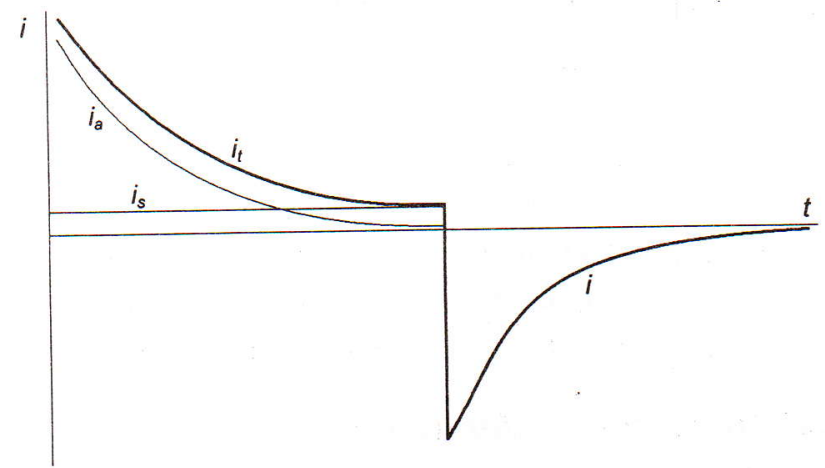

Fig. 2: Total current during charging and discharging 
Geometrical capacity current is so quick (about $10^{-12} \mathrm{~s}$ ) that it cannot be neglected. Then the total current can be described as:

$$
i_{t}(t)=i_{s}+i_{a}(t)
$$

For a macroscopic description of current responses the equivalent Maxwell - Wagner model can be used for dielectric materials. The equivalent model of the insulating material shown in Fig. 3 is based on $n$ independent Debye polarization processes. Each process has its own time constant of stabilization $\tau_{i}$ and maximum of elementary current $I_{m i}$, and by observing its changes we can obtain information about the state of the insulation system.

$$
i_{t}(t)=i_{s}+\sum_{i=1}^{n} I_{m i} \exp \left(\frac{-l}{\tau_{i}}\right)
$$

The values of the $R$ and $C$ elements of the model are calculated from the observed absorptive or resorptive currents. They represent time independent elements - steady current $i_{s}$ and time dependent polarization element $i_{a}(t)$. The principle of analysis is described by following equations:

$$
\begin{gathered}
i_{s}=\frac{U_{0}}{R_{0}} \\
I_{m i}=\frac{U_{i}}{R_{i}} \\
\tau_{i}=R_{i} \cdot C_{i}
\end{gathered}
$$

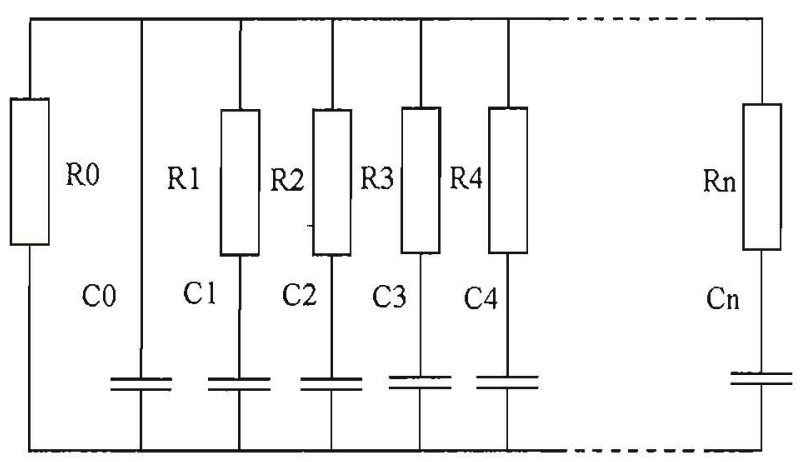

Fig. 3: Maxwell - Wagner equivalent model for dielectric materials

In the past the polarization spectrum of an insulation material was scanned only by polarization indices. These are described very easily as a ratio of observing absorptive or resorptive currents at a determined time. One-minute and 10-minute polarization indices are normally used.

$$
\begin{aligned}
& p_{1}=\frac{I_{15}}{I_{60}} \\
& p_{10}=\frac{I_{60}}{I_{600}},
\end{aligned}
$$

where $I_{15} \quad$ magnitude of observing current at $15 \mathrm{~s}$,

$I_{60} \quad$ magnitude of observing current at $60 \mathrm{~s}$,

$I_{600} \quad$ magnitude of observing current at $600 \mathrm{~s}$.

\subsection{Microscopic exhibition}

Basic background information can be found in works of Simmons, Tamm and Ewers [6]. The probability of the emis- sion of negative charges from the trapping level with activation energy

$$
W_{T}=W_{L}-W_{H}
$$

$W_{\downarrow} \quad$ energy of low conduction level,

$W_{T} \quad$ activation energy for charge emission from trapping level,

$W_{H} \quad$ energy of trapping level.

The observing current for a surface unit is

$$
i_{N}(t) \cdot t=\frac{q \cdot d \cdot k \cdot T}{2} \cdot \Gamma(W) \cdot V(W)
$$

$\Gamma(W)$ initialization of occupation of trapping level,

$V(W)$ energy spectrum of trapping levels,

$q$ charge,

$d$ thickness of sample,

$k$ Boltzmann's constant,

$T$ temperature.

$W_{H}$ is time dependent because while occupying the energy level it retreats from the conducting level. $W_{\gamma}(l)$ is directly proportional to time.

$$
\begin{gathered}
W_{T}=W_{L}-W_{H}=k \cdot T \cdot \ln (v \cdot t) \\
\Gamma(W) \cdot V(W)=\frac{2}{q \cdot d \cdot k \cdot T} \cdot t \cdot\left[I_{0}+\sum_{i=1}^{n} a_{i} \cdot \mathrm{e}^{-\frac{1}{\tau_{i}}}\right]
\end{gathered}
$$

As we can see, the result is equation (12) with the steady state element in brackets $I_{0}$ that represents the steady current $i_{s}$ from equation (3) or element $\frac{U_{0}}{R_{0}}$ from equation (4). Element $\sum_{i=1}^{n} a_{i} \cdot \mathrm{e}^{-t_{i}}$ represents the absorption current $i_{a}(t)$ from equation (2) or it is identical with the element from equation (3).

The conclusion is that macroscopic and microscopic views of the polarization processes when applying direct voltage lead to the same results.

\section{Influence of steady current}

Calculating the stabilization times of Debye independent polarization processes is one way to test the state of the insulation system. Another way to observe the polarization spectrum is transform the current response to the frequency domain.

The idea was to observe the influence of the steady element in the polarization current on two occasions. First, there were the modeling data. If the data is prepared in an artificial mode, the exact value of any element from equation (3) is known. This enables a correct comparison of the results obtained from calculations with the artificial input data [7]. The data sets were prepared with and without the steady element, and the calculations were also done with two modes - calculation when the steady elements were present and also when they were not present.

Table 1 shows the components of the artificial data. Seven elements were selected on the basis of our knowledge. For one set the steady element was added. 
Table 1: Components of artificial data

\begin{tabular}{|c|c|c|c|c|c|c|c|c|c|c|}
\hline & & $i$-th element & steady & 1 & 2 & 3 & 4 & 5 & 6 & 7 \\
\hline \multirow{2}{*}{ Model 0} & \multirow{2}{*}{ without steady } & $\tau_{i}[\mathrm{~s}]$ & & 0.1 & 0.5 & 1 & 5 & 10 & 50 & 100 \\
\hline & & $I_{m i}[\mathrm{pA}]$ & 0 & 100000 & 50000 & 10000 & 5000 & 1000 & 500 & 100 \\
\hline \multirow{2}{*}{ Model 1} & \multirow{2}{*}{ with steady } & $\tau_{i}[\mathrm{~s}]$ & & 0.1 & 0.5 & 1 & 5 & 10 & 50 & 100 \\
\hline & & $I_{m i}[\mathrm{pA}]$ & 10 & 100000 & 50000 & 10000 & 5000 & 1000 & 500 & 100 \\
\hline
\end{tabular}

As is shown in Table 1 , model 1 is the same as model 0 , except that the steady element is added for amplitude $10 \mathrm{pA}$. This allows a very strict distinction of differences between the two models as the influence of the DC component.

Both models were analyzed with two modes of calculation. First, without the presence of the DC component (model 0 and model 1) two data sets were achieved. Then the same mode of calculation was performed, but with the presence of the DC component.

It is not easy to perform the calculation. It is virtually impossible to calculate seven elements directly. The computation process is very unstable and it is very sensitive to the initial conditions. A better way is to compute only one element first. This means that the total current will be replaced by a single polarization process. This process is represented by one element from equation (3), where parameter $n=1$. DC elements may or may not be present. Then another element is added and the calculation is repeated. The result

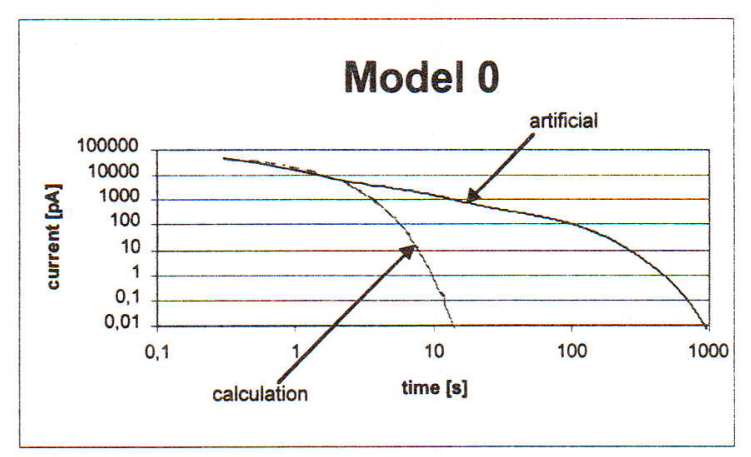

Fig. 4: One element

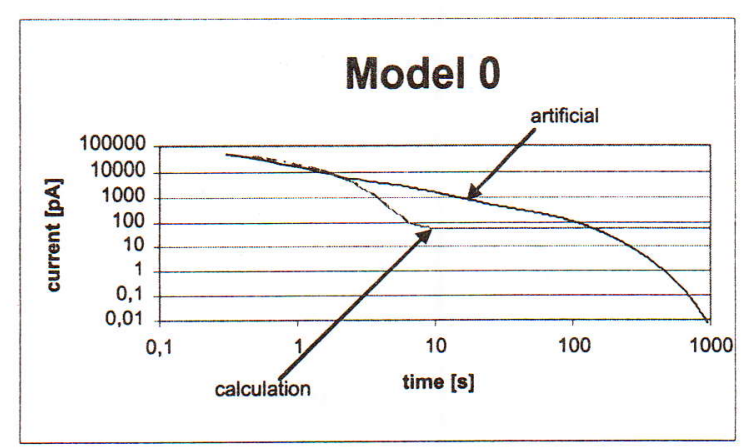

Fig. 6: One element replaces the total current with two elementary polarization processes (in the macroscopic mode there are two elementary current responses). Then more elements are added until all seven elements are used in the calculation.

The results are shown in Fig. 4 . It shows the data for model 0 and also the artificial data. There is only one elementary process in the calculation. The calculation is done without the steady element. There are large differences between them. Fig. 5 shows the same situation, except that the seven polarization processes are present in the computation. It is hard to distinguish between the artificial data and the data achieved from modeling according to equation (3).

The situation is practically the same when the steady element is present in the calculation.

For one element the error is large, and for seven elements the calculation and the artificial data are the same. The conclusion is evident and no more results are needed.

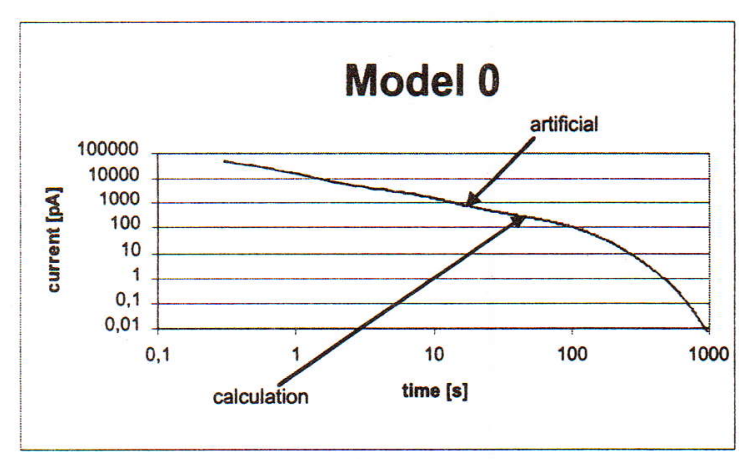

Fig. 5: Seven elements

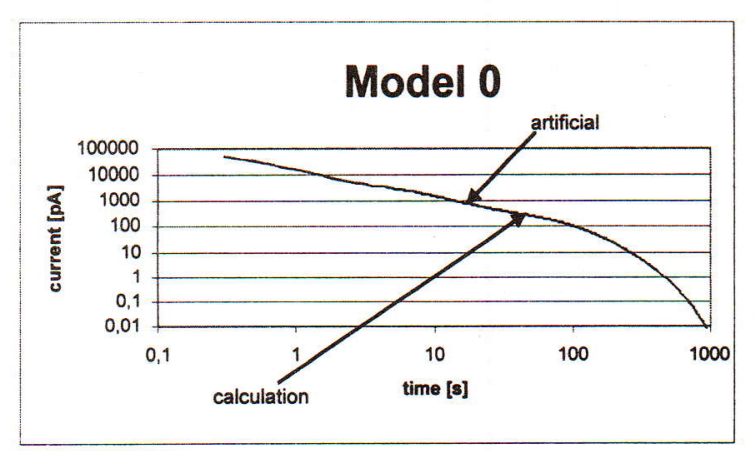

Fig. 7: Seven elements 
Table 2: Results of the computation

\begin{tabular}{|c|c|c|c|c|c|c|c|}
\hline \multirow{2}{*}{$\begin{array}{c}\text { No. of } \\
\text { elements }\end{array}$} & \multirow{2}{*}{ Element } & \multicolumn{2}{|c|}{ Model 0} & \multicolumn{2}{|c|}{ Model 1} & \multicolumn{2}{|c|}{ Real cable } \\
\hline & & With steady & Without steady & With steady & Without steady & With steady & Without steady \\
\hline \multirow{3}{*}{1} & $I_{m \mathrm{I}}$ & 59408 & 59239 & 59408 & 59207 & 891.00 & 290 \\
\hline & $I_{0}$ & 54 & & 64 & & 223.00 & \\
\hline & $\tau_{1}$ & 0.87 & 0.88 & 0.87 & 0.88 & 13.56 & 2281.65 \\
\hline \multirow{5}{*}{2} & $I_{m 1}$ & 69528 & 69441 & 69528 & 69401 & 1112.00 & 922 \\
\hline & $I_{m 2}$ & 6030 & 5927 & 6030 & 5880 & 406.00 & 241 \\
\hline & $I_{0}$ & 22 & & 32 & & 220.00 & \\
\hline & $\tau_{1}$ & 0.53 & 0.54 & 0.53 & 0.54 & 2.78 & 11.86 \\
\hline & $\tau_{2}$ & 8.06 & 8.33 & 8.06 & 8.47 & 30.66 & 7112.8 \\
\hline \multirow{7}{*}{3} & $I_{m 1}$ & 70462 & 70461 & 70462 & 70454 & 1094.00 & 1110 \\
\hline & $I_{m 2}$ & 7861 & 7841 & 7861 & 7775 & 443.00 & 427 \\
\hline & $I_{m 3}$ & 876 & 857 & 876 & 800 & 63.00 & 228 \\
\hline & $I_{0}$ & 3 & & 13 & & 218.00 & \\
\hline & $\tau_{1}$ & 0.48 & 0.48 & 0.48 & 0.48 & 2.26 & 2.59 \\
\hline & $\tau_{2}$ & 4.26 & 4.3 & 4.26 & 4.43 & 20.04 & 27.43 \\
\hline & $\tau_{3}$ & 43.13 & 44.64 & 43.13 & 49.88 & 97.41 & 16979.8 \\
\hline \multirow{9}{*}{4} & $I_{m !}$ & 48763 & 48748 & 48763 & 48740 & 1016.00 & 1066 \\
\hline & $I_{m 2}$ & 45562 & 45411 & 45562 & 44182 & 316.00 & 380 \\
\hline & $I_{m 3}$ & 5996 & 5987 & 5996 & 5915 & 303.00 & 178 \\
\hline & $I_{m 4}$ & 659 & 653 & 659 & 609 & 20.00 & 224 \\
\hline & $I_{0}$ & 1 & & 11 & & 217.00 & \\
\hline & $\tau_{1}$ & 0.2 & 0.2 & 0.2 & 0.21 & 1.89 & 2.06 \\
\hline & $\tau_{2}$ & 0.66 & 0.66 & 0.66 & 0.67 & 8.74 & 13.93 \\
\hline & $\tau_{3}$ & 5.6 & 5.63 & 5.6 & 5.8 & 31.48 & 46.24 \\
\hline & $\tau_{4}$ & 53.81 & 54.7 & 53.81 & 61.87 & 233.06 & 27466.08 \\
\hline \multirow{11}{*}{5} & $I_{m l}$ & 58560 & 57487 & 58560 & 48829 & 908.00 & 1018 \\
\hline & $I_{m 2}$ & 52723 & 52505 & 52723 & 46075 & 319.00 & 278 \\
\hline & $I_{m 3}$ & 3778 & 3882 & 3778 & 6025 & 320.00 & 311 \\
\hline & $I_{m 4}$ & 4010 & 3759 & 4010 & 677 & 113.00 & 39 \\
\hline & $1 \mathrm{~m}_{5}$ & 580 & 571 & 580 & 21 & 13.00 & 222 \\
\hline & $I_{0}$ & 1 & & 11 & & 216.00 & \\
\hline & $\tau_{1}$ & 0.14 & 0.14 & 0.14 & 0.2 & 1.71 & 1.91 \\
\hline & $\tau_{2}$ & 0.58 & 0.58 & 0.58 & 0.65 & 5.01 & 8.35 \\
\hline & $\tau_{3}$ & 2.63 & 2.82 & 2.63 & 5.52 & 20.18 & 27.41 \\
\hline & $\tau_{4}$ & 7.02 & 7.23 & 7.02 & 50.3 & 49.53 & 94.51 \\
\hline & $\tau_{5}$ & 58.82 & 59.8 & 58.82 & 965.32 & 389.13 & 39793.24 \\
\hline
\end{tabular}




\begin{tabular}{|c|c|c|c|c|c|c|c|}
\hline \multirow{2}{*}{$\begin{array}{c}\text { No. of } \\
\text { elements }\end{array}$} & \multirow{2}{*}{ Element } & \multicolumn{2}{|c|}{ Model 0} & \multicolumn{2}{|c|}{ Model 1} & \multicolumn{2}{|c|}{ Real cable } \\
\hline & & With steady & Without steady & With steady & Without steady & With steady & Without steady \\
\hline \multirow{13}{*}{6} & $I_{m \mathrm{l}}$ & 127229 & 84099 & 127229 & 58626 & & \\
\hline & $I_{m 2}$ & 50355 & 52594 & 50355 & 52742 & & \\
\hline & $I_{m 3}$ & 10631 & 6551 & 10631 & 3764 & & \\
\hline & $I_{m 4}$ & 5532 & 5425 & 5532 & 4043 & & \\
\hline & $I_{m 5}$ & 451 & 400 & 451 & 581 & & \\
\hline & $I_{m 6}$ & 460 & 407 & 460 & 11 & & \\
\hline & $I_{0}$ & 0 & & 10 & & & \\
\hline & $\tau_{1}$ & 0.09 & 0.11 & 0.09 & 0.14 & & \\
\hline & $\tau_{2}$ & 0.49 & 0.52 & 0.49 & 0.58 & & \\
\hline & $\tau_{3}$ & 1.01 & 1.23 & 1.01 & 2.61 & & \\
\hline & $\tau_{4}$ & 5.44 & 5.66 & 5.44 & 6.99 & & \\
\hline & $\tau_{5}$ & 19.46 & 24.84 & 19.46 & 58.58 & & \\
\hline & $\tau_{6}$ & 66.14 & 69.64 & 66.14 & 18579.76 & & \\
\hline \multirow{15}{*}{7} & $I_{m 1}$ & 98535 & 100003 & 98535 & 91222 & & \\
\hline & $I_{m 2}$ & 50309 & 50000 & 50309 & 52003 & & \\
\hline & $I_{m 3}$ & 9625 & 10000 & 9625 & 8415 & & \\
\hline & $I_{m 4}$ & 5074 & 5000 & 5074 & 5434 & & \\
\hline & $I_{m 5}$ & 895 & 1000 & 895 & 577 & & \\
\hline & $I_{m 6}$ & 496 & 500 & 496 & 104 & & \\
\hline & $I_{m 7}$ & 102 & 100 & 102 & 10 & & \\
\hline & $I_{0}$ & 0 & & 10 & & & \\
\hline & $\tau_{1}$ & 0.1 & 0.1 & 0.1 & 0.1 & & \\
\hline & $\tau_{2}$ & 0.5 & 0.5 & 0.5 & 0.51 & & \\
\hline & $\tau_{3}$ & 1.02 & 1 & 1.02 & 1.16 & & \\
\hline & $\tau_{4}$ & 5.07 & 5 & 5.07 & 5.89 & & \\
\hline & $\tau_{5}$ & 10.36 & 10 & 10.36 & 44.92 & & \\
\hline & $\tau_{6}$ & 50.12 & 50 & 50.12 & 107.03 & & \\
\hline & $\tau_{7}$ & 99.5 & 100 & 99.5 & 268880 & & \\
\hline
\end{tabular}

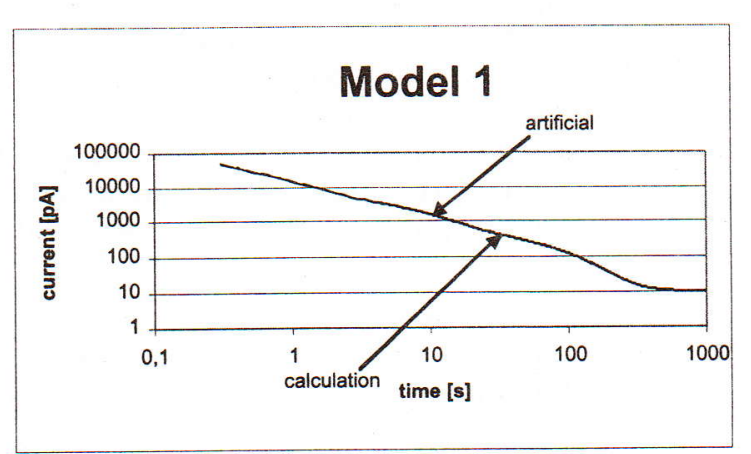

Fig. 8: Calculation without steady element

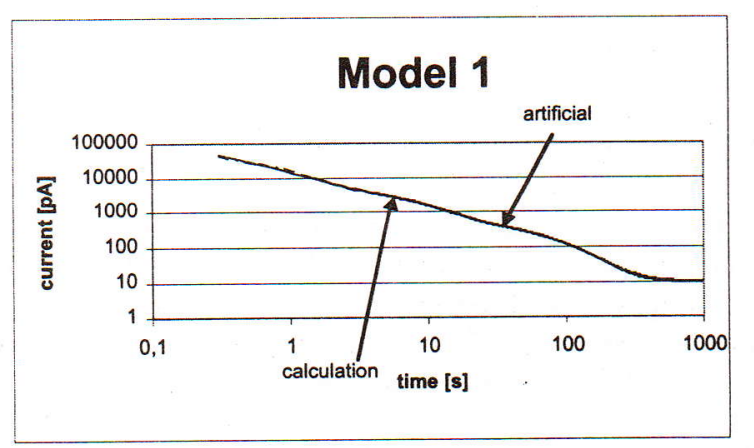

Fig. 9: Calculation with steady element 
Let us have a look at model 0 and the force calculation with the steady element (the first two columns of data) with expansion to seven elementary polarization processes (number of elements - 7). It can be seen that the calculation is quite successful. Only small differences appear between the artificial and calculated data. When the calculation was done without the steady component, the results exactly match with the artificial data. The conclusion is that the calculation really reveals the presence of the steady element, and its value can also be defined.

In the case of model 1 (artificial data with a steady element of magnitude $10 \mathrm{pA}$ ), the calculation with obligatory presence of the steady component is also successful. In the third column of data with 7 expansion elements can be seen that the calculation exactly revealed steady element $I_{0}$ and determined its value. The forth column belongs to calculation without forced of steady component. The results are completely wrong. The time constants and amplitudes of the elementary polarization processes have large errors. This leads to the conclusion that if the steady element is present and the calculation does not require it, the result is completely wrong.

This situation was as expected. The calculation can reveal the steady element, and it is recommended to required calculation with it. If the element is present, the calculation reveals it. If the element is not present and the calculation is required to compute it, the result is a steady element with a magnitude of 0 .

The final situation was examined by measuring of real object. The cable was examined after accelerated thermal stress for a period of 7000 hours at a temperature of $100^{\circ} \mathrm{C}$. The cable was made from XPLE and for a $22 \mathrm{kV}$ voltage operating level. The cable was charged with direct voltage at $100 \mathrm{~V}$ and the current response was observed for a period of $1000 \mathrm{~s}$. The observing time was selected to have the same time window of polarization processes as in the artificial data for model 0 and model 1. Fig. 10 shows the measuring set.

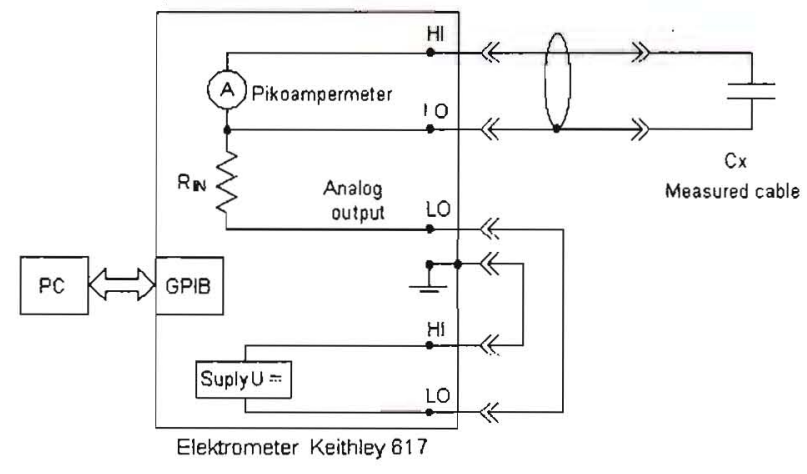

Fig. 10: Measured equipment for cable

The electrometer can measure currents down to $10^{-16} \mathrm{~A}$ and it is suitable for our purposes. Fig. 11 shows the measured cable response and the calculation without required steady element. The values of the data are given in the fifth column of data in Table 2. Fig. 12 shows the same situation but without a required steady component. The situations in the data values are completely changed. Although the data in graphic form seems to be the same, the magnitudes are different. The charging time was $1000 \mathrm{~s}$ and for this reason the observing time constant $\tau_{i}$ cannot be higher than 10 -times. The reason for this is based on the physical background. If the charging lasts $1000 \mathrm{~s}$, polarization processes with a level no higher than 10-times the time duration can be excited. Nevertheless the calculations only to five elementary processes were successful. No polarizations with a higher time constant were started.

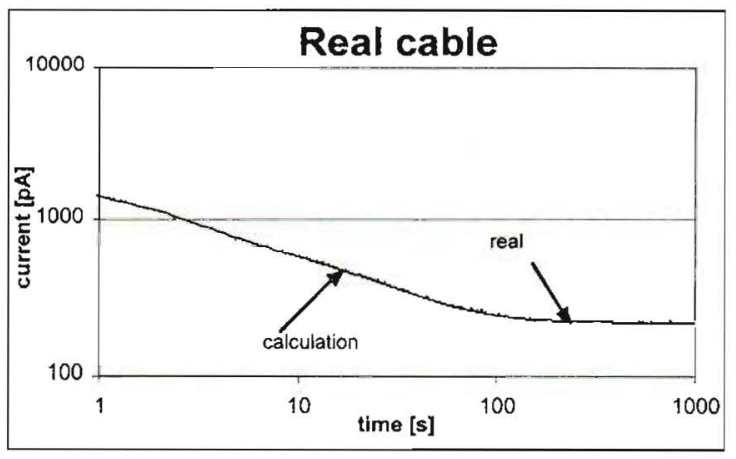

Fig. 11: Calculation without steady element

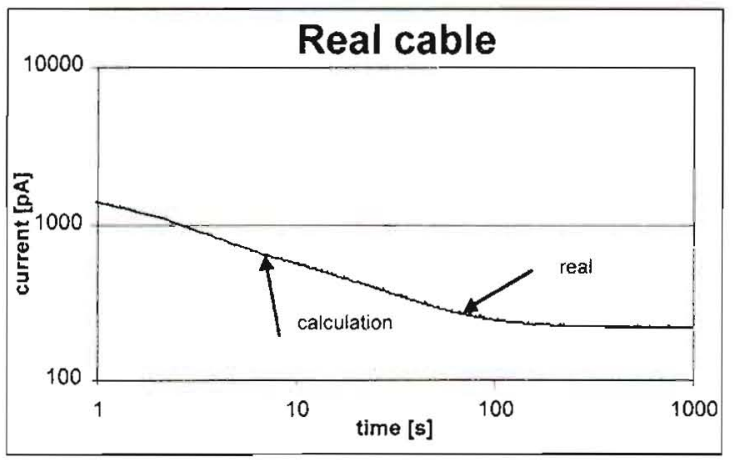

Fig. 12: Calculation with steady element

\section{Conclusion}

This paper deals with calculating elements of equivalent models for dielectric materials. The basic principles of the methods were described, together with the magnitudes of the elementary polarization currents and the time constant of their stabilization. First of all, the artificial data was verified. The calculation was to the presence of a steady element.

Then the approximation of real data was carried out successfully. It was demonstrated, that graphical fitting alone does not allow to claim that a model of a real object has been achieved. The magnitudes of the observing elements have to be investigated with their electro-physical background. Although higher values were calculated and graphical fitting confirmed them, the physical processes did not start and they did not really exist.

The real object has a direct element because it is at the end of its life after long-term accelerated thermal stress [8]. This is evident from graphical representation of real measured data. For this reason calculation with a required steady element must always be made, whether this steady component really exists or not. 


\section{References}

[1] Németh, E., Horváth, T.: Fundamentals of the Stimulation of Dielectric Processes of Insulations. $8^{\text {th }}$ ISH, Yokohama, Japan 1993.

[2] Cimbala, R.: Diagnostic Method of Insulation Systems with Ultra-low Frequencies Utilization. (In Slovak). $5^{\text {th }}$ Science Conference EF VŠT, Košice 1989, p. 169-173.

[3] Cimbala, R.: Current Response in Multilayer Insulation at Ultra-low Frequencies. (In Slovak). PhD Thesis, Department of High Voltage Engineering, Faculty of Electrical Engineering, Technical University Košice, Košice 1993.

[4] Liederman, K.: Dielectric Relaxation Spectroscopy as a Tool for Monitoring Degradation in PETP Foils. $8^{\text {th }}$ Science Conference FEI TU Košice 1996, p. 72-75.

[5] Kaláb, K.: The Possibilities of DC Diagnostics of Electrical Insulation. (In Czech). Science Conference Electro '95, Žilina 1995, p. 58-63.

[6] Simmons, J. G., Tam, H. C.: Theory of Isothermal Relaxation Current and the Direct Determination of Trap Parameters in Semiconductors and Insulations Containing Arbitrary. Physical review B, Vol. 7, 1973, No. 8.
[7] Záliš, K.: Database System for Prophylactic Measurement Processing on hv Alternators. (In Czech). Science Conference Diagnostika '93, Plzeň 1993, p. 44-48.

[8] Šandrik, P., Zlatovský, J., Sabela, P.: Development of Diagnostic Parameters during Life-time of Insulation Systems. (In Slovak). $8^{\text {th }}$ Science Conference FEI TU, Košice 1996 , p. 79-82.

Assoc. prof. Ing. Roman Cimbala, Ph.D.

phone/fax : +421556225060

e-mail: cimbala@ktvnl.tuke.sk

Assoc. prof. Ing. Igor Kršňák

e-mail:krsnak@ktvn1.tuke.sk

Assoc. prof. Ing. Iraida Kolcunová, Ph.D.

e-mail:iraida@ktvnl.tuke.sk

Department of High Voltage Engineering

Technical University Košice

Faculty of Electrical Engineering and Informatics Mäsiarska 74

04001 Košice, Slovak Republic 